\title{
Enfermedad por aglutininas frías: proceso diagnóstico, tratamiento y seguimiento de un paciente
}

\section{Cold agglutinin disease: diagnosis, treatment and follow-up of a patient}

\author{
Kenny Mauricio Gálvez-Cárdenas ${ }^{1}$ (D), Juan Felipe Morantes-Rubiano² (D, \\ Andrés Felipe Lotero-Cadavid ${ }^{3}$ (iD
}

\begin{abstract}
Resumen. La enfermedad por crioaglutininas es una anemia hemolítica autoinmune que se caracteriza, en la gran mayoría de los casos, por la hemólisis mediada por autoanticuerpos de tipo IgM y complemento C3d, contra los antígenos de la membrana del eritrocito, que conduce a hemólisis extravascular con propensión a la trombosis, y que afecta principalmente al sexo femenino y personas mayores. Su diagnóstico se realiza con la prueba de Coombs directo y fraccionado, y la titulación de aglutininas frías $>1: 64$ a $4{ }^{\circ} \mathrm{C}$. Se describe el caso clínico de una mujer de 89 años con un síndrome constitucional y una anemia de 3 años de evolución, en quien se determinó el diagnóstico de enfermedad por aglutininas frías. Asimismo, se describe el abordaje diagnóstico, el tratamiento instaurado, y se hace una breve revisión de la literatura publicada.
\end{abstract}

Palabras clave: anemia hemolítica autoinmune, enfermedad por crioaglutininas, complemento, livedo reticular, Raynaud, Coombs, rituximab.

Abstract. Cold agglutinin disease (CAD) is an autoimmune hemolytic anemia characterized in the vast majority of cases by hemolysis mediated by lgM autoantibodies and complement C3d against erythrocyte membrane antigens, leading to extravascular hemolysis with propensity to thrombosis, affecting mainly females and older individuals. It is diagnosed by direct and fractionated Coombs test and a cold agglutinin titer $>1: 64$ at $4{ }^{\circ} \mathrm{C}$. We describe the case of an 89-year-old woman with a constitutional syndrome and a 3-year history of anemia, who was diagnosed with cold agglutinin disease. Also, we include the diagnostic and treatment approach, and a brief review of the literature.

\footnotetext{
${ }^{1}$ Médico, Especialista en Medicina Interna y Hematología, Hospital Pablo Tobón Uribe. Medellín, Colombia.

${ }^{2}$ Médico, Universidad de La Sabana. Chía, Colombia. E-mail: morantesrubiano@hotmail.com.

${ }^{3}$ Médico, Especialista en Medicina Interna, Universidad Pontificia Bolivariana. Medellín, Colombia.

Conflicto de interés: los autores declaran que no tienen conflicto de interés.

Medicina \& Laboratorio 2021;25:735-742. https://doi.org/10.36384/01232576.527.

Recibido el 10 de julio de 2021; aceptado el 9 de septiembre de 2021. Editora Médica Colombiana S.A., 2021
} 
Keywords: hemolytic autoimmune anemia, cold agglutinin disease, complement, livedo reticularis, Raynaud, Coombs, rituximab.

\section{Introducción}

Las anemias hemolíticas autoinmunes son un grupo heterogéneo y poco común de enfermedades, que se caracterizan por la hemólisis mediada por autoanticuerpos contra los antígenos de la membrana del eritrocito [1,2]. Serológicamente y según la reactividad de los anticuerpos, se han clasificado como de tipo caliente que corresponde a un $60 \%$ a $70 \%$ de los casos, de tipo frío a un $20 \%$ a $25 \%$, y mixto al $1 \%$ a $5 \%$ de los casos restantes $[3,4]$. Por su parte, la anemia hemolítica autoinmune por anticuerpos fríos se categoriza como enfermedad por aglutininas frías (CAD, del inglés, Cold Agglutinin Disease), que corresponde al trastorno primario; hemoglobinuria paroxística fría, y síndrome de aglutininas frías (CAS, del inglés Cold Agglutinin Syndrome), secundario a procesos infecciosos (incluido COVID-19) [5,6], autoinmunes o malignidad [2,7-9].

A continuación, se presenta el caso de una paciente con $C A D$, una enfermedad rara en nuestro medio. Se hace un abordaje clínico a partir de la identificación de la anemia hemolítica autoinmune, con los estudios para la diferenciación de causas secundarias, y se describe el tratamiento indicado con el seguimiento a 1 año, para finalmente realizar una breve revisión de la literatura sobre esta patología poco común.

\section{Caso clínico}

Paciente femenina de 89 años, ama de casa, natural y procedente de Mede- llín, quien consultó por un cuadro clínico de aproximadamente 3 años de evolución, caracterizado por astenia, adinamia, dolor óseo y pérdida importante de peso; es clara en que hubo un periodo de tiempo de 3 meses donde presentó exacerbación de los síntomas y pérdida de hasta $20 \mathrm{~kg}$ de peso. Como antecedentes de importancia relató diabetes mellitus tipo 2 y enfermedad arterial oclusiva crónica. De manera cotidiana utilizaba insulina glargina, linagliptina, acetaminofén, esomeprazol, metoprolol y trazodona, y como antecedente toxicológico reportó ex tabaquismo con un índice paquete año (IPA) de 20.

De igual manera, reportó que desde hacía varios años se había documentado en laboratorios una anemia que requirió múltiples transfusiones sanguíneas durante ese periodo, cuya etiología no había sido clara, a pesar de haberse realizado múltiples estudios, entre ellos una endoscopia de vías digestivas altas y colonoscopia, que se reportaron como normales.

En el examen físico se destacó únicamente una palidez cutánea marcada, sin ningún otro hallazgo de importancia. Había consultado al servicio de urgencias en la semana previa, donde requirió la transfusión de 2 unidades de glóbulos rojos, y se dio egreso con la orden de los paraclínicos cuyos resultados se presentan en la tabla 1. Se evidenció una anemia hemolítica autoinmune con base en los resultados del Coombs directo positivo +++ y reticulocitosis, con hemoglobina de $6,4 \mathrm{~g} / \mathrm{dL}$, además de leucocitosis 
asociada a linfocitosis, y presencia de eritroblastos y mielocitos, así como un fenómeno de rouleaux en el extendido de sangre periférica. Por la sospecha de un posible síndrome linfoproliferativo que explicase el fenómeno hemolítico autoinmune, se consideró la realización de un aspirado y biopsia de médula ósea ( $A B M O)$, además de un Coombs fraccionado para aclarar el tipo de anticuerpo involucrado. Igualmente, por el hallazgo de un deterioro en la función renal (creatinina al ingreso de 1,30 $\mathrm{mg} / \mathrm{dL}$ versus 0,89 $\mathrm{mg} / \mathrm{dL}$ durante el seguimiento), se estimó importante el estudio de una gammapatía monoclonal, por lo cual se solicitó electroforesis de proteínas, inmunofijación y cadenas ligeras libres en suero (tabla 1 y figura 1).

Los estudios también reportaron un Coombs fraccionado con IgG y C3d positivo, por lo cual se solicitaron crioaglutininas, las cuales fueron positivas en $>1: 64$ diluciones (tabla 1). El ABMO reportó hallazgos sugestivos de un desorden linfoproliferativo crónico

Tabla 1. Paraclínicos de la paciente al ingreso y durante el seguimiento

\begin{tabular}{|c|c|c|}
\hline Parámetro & Ingreso & Seguimiento \\
\hline Hemoblobina (g/dL) & 6,4 & 10,8 \\
\hline Hematocrito (\%) & 18 & 31,4 \\
\hline $\operatorname{VCM}(f L)$ & 108,4 & 86,1 \\
\hline $\begin{array}{l}\text { Ancho de distribución } \\
\text { eritrocitaria (\%) }\end{array}$ & 17,9 & - \\
\hline Leucocitos (células $/ \mathrm{mm}^{3}$ ) & 11.400 & 4.200 \\
\hline Neutrófilos (células $/ \mathrm{mm}^{3}$ ) & 1.700 & 2.300 \\
\hline Linfocitos (células/mm³) & 8.500 & 800 \\
\hline Plaquetas (células $/ \mathrm{mm}^{3}$ ) & 139.000 & 213.000 \\
\hline $\begin{array}{l}\text { Recuento de reticulocitos } \\
(\%)\end{array}$ & 5,4 & 3 \\
\hline $\begin{array}{l}\text { Extendido de sangre } \\
\text { periférica }\end{array}$ & Fenómeno de rouleaux & Normal \\
\hline Coombs directo & +++ & Negativo \\
\hline Coombs fraccionado & $\operatorname{lgG}++; C 3 b / C 3 d+++$ & Negativo \\
\hline $\begin{array}{l}\text { Lactato deshidrogenasa } \\
\text { (U/L) }\end{array}$ & 257 & 189 \\
\hline Bilirrubina directa (mg/dL) & 0,29 & - \\
\hline Bilirrubina total (mg/dL) & 1,38 & - \\
\hline Haptoglobina (mg/dL) & 72 & 181 \\
\hline Creatinina (mg/dL) & 1,3 & 0,89 \\
\hline
\end{tabular}




\begin{tabular}{|c|c|c|}
\hline $\mathrm{BUN}(\mathrm{mg} / \mathrm{dL})$ & 48,3 & 10,2 \\
\hline Electroforesis de proteínas & $\begin{array}{l}\text { Dos bandas tenues en región } \\
\text { gamma }\end{array}$ & $\begin{array}{l}\text { Ligera banda tenue en región } \\
\text { gamma }\end{array}$ \\
\hline Inmunofijación en suero & $\begin{array}{l}\text { Patrón poligoclonal lgG, IgA, lgM, } \\
\text { kappa/lambda }\end{array}$ & $\begin{array}{l}\text { Gammapatía monoclonal } \\
\text { lgG kappa asociada a un } \\
\text { componente monoclonal } \\
\quad \text { lambda }\end{array}$ \\
\hline Crioaglutininas & Positivas $>1: 64$ diluciones & - \\
\hline Ferritina $(\mathrm{ng} / \mathrm{mL})$ & 924 & - \\
\hline Saturación de ferritina (\%) & 105 & - \\
\hline Transferrina (mg/dL) & 159 & - \\
\hline Vitamina B12 (pg/mL) & 510 & - \\
\hline \multicolumn{3}{|c|}{ Estudios de histopatología en médula ósea } \\
\hline \multicolumn{3}{|c|}{$\begin{array}{l}\text { Por la correlación entre los hallazgos de la biopsia de médula ósea, el patrón de inmunohistoquímica } \\
\text { (CD20+, coloración para ciclina D1 negativa), y el estudio de citometría de flujo (CD5-, CD10-), se } \\
\text { consideró que se trataba de un compromiso por un desorden linfoproliferativo crónico B asociado } \\
\text { a aglutininas frías. El fenotipo no era el característico de una leucemia linfocítica crónica, ni el de un } \\
\text { linfoma linfoplasmocítico. }\end{array}$} \\
\hline
\end{tabular}

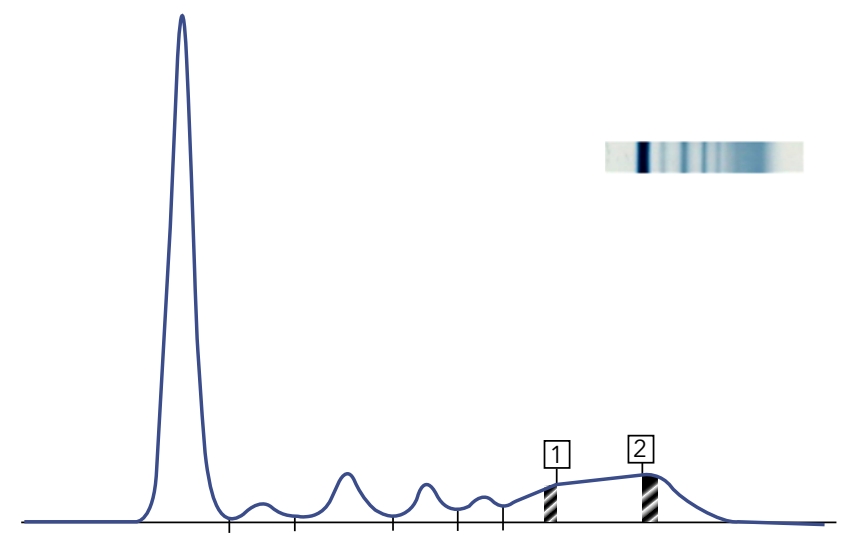

Figura 1. Electroforesis de proteínas del ingreso, donde se observan dos bandas tenues en la región gamma.

$(C D 20+)($ tabla 1), en tanto que los estudios para enfermedades infecciosas $y$ autoinmunes fueron negativos. En imágenes de cuello, tórax y abdomen no hubo evidencia de malignidad, de manera que se realizó el diagnóstico de enfermedad por aglutininas frías.
Se inició tratamiento con rituximab, anticuerpo monoclonal anti-CD20 a dosis de $375 \mathrm{mg} / \mathrm{m}^{2}$, con premedicación de acetaminofén, difenhidramina e hidrocortisona. La primera dosis se administró de manera intrahospitalaria, y las otras tres de forma ambulatoria. Luego 
de cumplir el esquema de cuatro dosis con rituximab, asistió a consulta externa, encontrándose una notable mejoría clínica reportada por la paciente y por los laboratorios de control (tabla 1), que evidenciaron un aumento considerable en los valores de hemoglobina, sin marcadores de hemólisis, con independencia transfusional y una negativización tanto del Coombs directo como del fraccionado.

\section{Discusión}

La CAD tiene una prevalencia de 5 a 20 casos por millón y una incidencia de 0,5 a 1,9 casos por millón por año, mostrando una variación considerable con el clima, y afecta predominantemente a personas de sexo femenino, mayores, pero puede ocurrir en personas más jóvenes [10-12]. En 2019, se publicó en Colombia la primera serie de casos por aglutininas frías, en donde se reportó que la edad promedio fue de 60 años, con el $82,5 \%$ de sexo femenino, características similares a las de otras series descritas en la literatura. Asimismo, se informó en esa serie que de 23 pacientes había 6 formas primarias, 4 asociadas con linfoma no Hodgkin (LNH), 8 secundarias a enfermedades infecciosas y autoinmunes, y 5 asociadas con enfermedades misceláneas [13].

La fisiopatología se relaciona con la presencia de autoanticuerpos monoclonales denominados crioaglutininas, por lo general de tipo lgM [10,14], que se unen a la superficie eritrocitaria en su paso a través de la circulación acral, donde hay un enfriamiento de la sangre, con una consecuente aglutinación. Las crioaglutininas permiten la unión de la proteína del complemento 1 (C1), con la iniciación de la vía clásica del complemento, donde la esterasa C1 activa C4 y C2, generando la C3 con- vertasa, que promueve la formación y cubrimiento eritrocitario con $\mathrm{C} 3 \mathrm{~b}$. Con el regreso de los glóbulos rojos a la circulación central (donde hay una mayor temperatura), se produce una liberación de la unión entre las crioaglutininas y la membrana celular, lo que conduce a un cese de la aglutinación, sin embargo, persistirá la unión de C3b con la célula $[7,15]$. Una proporción de los glóbulos rojos serán secuestrados por macrófagos en el sistema reticuloendotelial, produciendo la destrucción extravascular de eritrocitos, lo que explica el mecanismo principal de hemólisis en el paciente estable, no obstante, las células restantes sufrirán otro proceso de escisión en la C3b, dejando C3d unido en la membrana (hallazgo común en pruebas de laboratorio), pudiendo conducir a la activación de C5 y a la formación del complejo de ataque a la membrana, con una subsecuente hemólisis intravascular, presente principalmente en escenarios de exacerbación de la enfermedad [7,16].

Por lo general, el diagnóstico se enmarca en el estudio de una anemia crónica de origen indeterminado y de grado variable, usualmente en pacientes ancianos, con manifestaciones clínicas asociadas a la fisiopatología de aglutinación acral, en la que se incluye la presencia de livedo reticular [17], fenómeno de Raynaud, acrocianosis y en casos raros, necrosis cutánea. La presencia de estos síntomas fríos varía en la literatura, desde un $90 \%$ [18] hasta un $39 \%$ en otras series de pacientes $[18,19]$.

Los hallazgos en los exámenes de laboratorio que orientan el diagnóstico son anemia hemolítica con reticulocitosis, hiperbilirrubinemia, lactato deshidrogenasa elevada, haptoglobina disminuida, presencia en el frotis de sangre periférica de eritrocitos agluti- 
nados, anisocitosis y policromasia [10]; por otro lado, es necesario una prueba de Coombs positiva para C3 y clásicamente negativa para lgG, sin embargo, se ha encontrado una positividad para C3d e lgG hasta en un 20\% de los pacientes $[3,4,19,20]$, como sucedió en el caso expuesto. Adicionalmente, se requieren unos títulos de aglutininas frías $>1: 64$, que son necesarios para el diagnóstico, y que estaban presentes en la paciente en cuestión.

Como criterios confirmatorios, pero no requeridos para el diagnóstico (tabla 2), se tiene la identificación por inmunohistoquímica de un desorden linfoproliferativo clonal en médula ósea, junto con la realización de electroforesis de proteínas e inmunofijación en suero para identificar desórdenes monoclonales. Finalmente, con relación al diagnóstico, es importante resaltar que siempre se deben estudiar y descartar causas secundarias, lo cual se puede hacer a partir de la realización de una historia clínica y un examen físico completo, apoyados en imágenes diagnósticas [19,21].

La mayoría de los pacientes presentan una anemia crónica que no requiere terapias más allá de la suplementación con folatos, y medidas orientadas a la protección de las extremidades contra el frío, además, deben evitar infusiones intravenosas frías en caso de necesitarlas. Sin embargo, pacientes con cuadros de mayor gravedad, quienes requieren transfusiones regulares, deben recibir tratamiento farmacológico orientado a la reducción de la producción de lgM, la cual es responsable de la fijación del complemento.

Tabla 2. Criterios diagnósticos para enfermedad por aglutininas frías $[7,19]$

\begin{tabular}{|c|c|c|}
\hline & Criterio & Procedimientos y aspectos claves \\
\hline \multirow{5}{*}{$\begin{array}{l}\text { Requerido para el } \\
\text { diagnóstico }\end{array}$} & Hemólisis crónica & \\
\hline & $\begin{array}{l}\text { Coombs poliespecífico (directo) } \\
\text { positivo }\end{array}$ & \\
\hline & $\begin{array}{l}\text { Coombs monoespecífico } \\
\text { (fraccionado) positivo para C3d }\end{array}$ & $\begin{array}{l}\text { Por lo general, es negativo para lgG, } \\
\text { pero ocasionalmente puede ser } \\
\text { levemente positivo }\end{array}$ \\
\hline & $\begin{array}{l}\text { Títulos de aglutininas frías }>1: 64 \\
\text { a } 4{ }^{\circ} \mathrm{C}\end{array}$ & $\begin{array}{l}\text { La muestra de sangre se debe } \\
\text { almacenar entre } 37^{\circ} \mathrm{C} \text { a } 38^{\circ} \mathrm{C} \text { hasta } \\
\text { que el suero sea removido del } \\
\text { coágulo }\end{array}$ \\
\hline & Sin malignidad subyacente & $\begin{array}{l}\text { Valoración clínica e imágenes } \\
\text { radiológicas }\end{array}$ \\
\hline \multirow{3}{*}{$\begin{array}{l}\text { Confirmatorio, pero } \\
\text { no requerido para el } \\
\text { diagnóstico }\end{array}$} & $\begin{array}{l}\text { IgM kappa monoclonal en suero; } \\
\text { rara vez } \lg G, \lg A \text { o fenotipo } \lambda\end{array}$ & $\begin{array}{l}\text { La muestra de sangre se debe } \\
\text { almacenar entre } 37^{\circ} \mathrm{C} \text { a } 38^{\circ} \mathrm{C} \text { hasta } \\
\text { que el suero sea removido del } \\
\text { coágulo }\end{array}$ \\
\hline & $\begin{array}{l}\text { Razón } k / \lambda \text { en población de células } \\
B>3,5 \text { (rara vez }<0,9)\end{array}$ & $\begin{array}{l}\text { Citometría de flujo en aspirado de } \\
\text { médula ósea }\end{array}$ \\
\hline & $\begin{array}{l}\text { Desorden linfoproliferativo } \\
\text { asociado por histología }\end{array}$ & Biopsia de médula ósea \\
\hline
\end{tabular}


Entre los esquemas de tratamiento propuestos para CAD se tiene la monoterapia con rituximab, con respuestas reportadas cercanas al $50 \%$ de los pacientes, pero no duraderas [22], por lo que se ha buscado asociarlo a otros fármacos como la fludarabina, alcanzándose una mayor respuesta hasta en el $76 \%$ de los pacientes que no presentaron respuesta a rituximab como primera línea $[11,21]$. No obstante, se debe resaltar la mayor toxicidad con el uso asociado de fludarabina, reportándose neutropenia y mayor incidencia de infecciones. También, se ha estudiado el uso conjunto de rituximab con bendamustina, que ha demostrado efectividad, logrando mayores tasas de respuesta completa, pudiendo establecerse como una terapia de primera línea, aunque también con riesgo de mielosupresión e infección, pero en menor proporción que con el uso de fludarabina [19].

Para determinar si la respuesta a la terapia indicada fue completa, parcial o sin respuesta, se evalúa con base en la mejoría clínica del cuadro, los niveles de hemoglobina, la ausencia de he- mólisis y otros parámetros establecidos en la tabla 3.

\section{Conclusión}

La CAD es una entidad clínico-patológica rara que se debe sospechar en pacientes ancianos con síntomas acrales inducidos por el frío, y en presencia de una anemia hemolítica. Se resalta la importancia de realizar un adecuado abordaje de la anemia hemolítica, identificando el tipo de anticuerpo involucrado en la hemólisis del eritrocito, tomando en cuenta que las muestras de sangre recolectadas mediante flebotomía se coagularán poco después de la exposición a la temperatura del ambiente, por lo que es vital mantener caliente el tubo de muestra durante la extracción de sangre y durante el transporte al laboratorio para una mejor precisión de las pruebas [23]. Se recomienda también el estudio completo de causas secundarias como enfermedades autoinmunes, infecciosas o neoplásicas para distinguir entre el CAD y el CAS, ya que de ello va a depender el tratamiento y la respuesta del paciente.

Tabla 3. Criterios de respuesta utilizados en ensayos clínicos para enfermedad por aglutininas frías $[7,19]$

\begin{tabular}{|c|c|}
\hline Nivel de respuesta & Definición \\
\hline Respuesta completa & $\begin{array}{l}\text { - Ausencia de anemia } \\
\text { - Sin signos de hemólisis } \\
\text { - Desaparición de síntomas de la CAD } \\
\text { - Ausencia de proteínas monoclonales en suero } \\
\text { - Ausencia de linfoproliferación clonal en histología de } \\
\text { médula ósea, inmunohistoquímica y citometría de flujo }\end{array}$ \\
\hline Respuesta parcial & $\begin{array}{l}\text { - Incremento estable de los niveles de hemoglobina de al } \\
\text { menos 2,0 } \mathrm{g} / \mathrm{dL} \text { o al rango de normalidad } \\
\text { - Reducción de los niveles séricos de } \lg M>50 \% \text { del nivel } \\
\text { basal o que alcancen valores de normalidad } \\
\text { - Mejoría de síntomas clínicos } \\
\text { - Independencia transfusional }\end{array}$ \\
\hline No respuesta & - Sin criterios de respuesta completa ni respuesta parcial \\
\hline
\end{tabular}




\section{Referencias}

1. Hill QA, Stamps R, Massey E, Grainger JD, Provan D, Hill A. The diagnosis and management of primary autoimmune haemolytic anaemia. Br J Haematol 2017;176:395-411. https:// doi.org/10.1111/bjh.14478.

2. Gelman R, Kharouf F, Ishay Y, Gural A. Cold agglutinin-mediated autoimmune hemolytic anemia in association with antiphospholipid syndrome. Acta Haematol 2021:1-4. https:// doi.org/10.1159/000516295.

3. Barcellini W, Fattizzo B. The changing landscape of autoimmune hemolytic anemia. Front Immunol 2020;11:946. https://doi.org/10.3389/ fimmu.2020.00946.

4. Jäger U, Barcellini W, Broome CM, Gertz MA, Hill A, Hill QA, et al. Diagnosis and treatment of autoimmune hemolytic anemia in adults: Recommendations from the First International Consensus Meeting. Blood Rev 2020;41:100648. https:// doi.org/10.1016/j.blre.2019.100648.

5. Kaur J, Mogulla S, Khan R, Krishnamoorthy G, Garg S. Transient cold agglutinins in a patient with COVID-19. Cureus 2021;13:e12751. https://doi.org/10.7759/cureus.12751.

6. Raghuwanshi B. Serological blood group discrepancy and cold agglutinin autoimmune hemolytic anemia associated with novel coronavirus. Cureus 2020;12:e11495. https://doi. org/10.7759/cureus.11495.

7. Berentsen S, Randen U, Tjønnfjord GE. Cold agglutinin-mediated autoimmune hemolytic anemia. Hematol Oncol Clin North Am 2015;29:455471. https://doi.org/10.1016/j.hoc.2015.01.002.

8. Gupta R, Singh S, Anusim N, Gupta S, Gupta S, Huben M, et al. Coronavirus disease 2019 and cold agglutinin syndrome: An interesting case. Eur J Case Rep Intern Med 2021;8:002387. https://doi.org/10.12890/2021_002387.

9. Brugnara C, Berentsen S. UptoDate. Cold agglutinin disease. Países bajos: Wolters Kluwer; 2021. Acceso 15 de junio de 2021. Disponible en https://www.uptodate.com/contents/cold-agglutinin-disease\#H3360841552.

10. Berentsen S, Malecka A, Randen U, Tjønnfjord GE. Cold agglutinin disease: where do we stand, and where are we going? Clin Adv Hematol Oncol 2020;18:35-44.
11. Berentsen S. How I manage patients with cold agglutinin disease. Br J Haematol 2018;181:320330. https://doi.org/10.1111/bjh.15109.

12. Broome CM. Diagnosis and treatment of cold agglutinin disease. Clin Adv Hematol Oncol 2019;17:162-165.

13. Atencia CJ, Quintero $\mathbf{C}$. Primera serie de casos reportada en Colombia de la enfermedad por aglutininas frías primaria y secundaria. latreia 2020;33:123-132. https://doi.org/10.17533/ udea.iatreia. 40

14. Berentsen S. How I treat cold agglutinin disease. Blood 2021;137:1295-1303. https://doi. org/10.1182/blood.2019003809.

15. Baines AC, Brodsky RA. Complementopathies. Blood Rev 2017;31:213-223. https:// doi.org/10.1016/j.blre.2017.02.003.

16. Berentsen $S$, Ulvestad $E$, Langholm $R$, Beiske K, Hjorth-Hansen $\mathbf{H}$, Ghanima $\mathbf{W}$, et al. Primary chronic cold agglutinin disease: a population based clinical study of 86 patients. Haematologica 2006;91:460-466.

17. Sharma K, Patel A. Livedo reticularis in cold agglutinin disease. N Engl J Med 2019;381:e27. https://doi.org/10.1056/NEJMicm1902289.

18. Swiecicki PL, Hegerova LT, Gertz MA. Cold agglutinin disease. Blood 2013;122:1114-1121. https://doi.org/10.1182/blood-2013-02-474437.

19. Berentsen S, Tjønnfjord GE. Diagnosis and treatment of cold agglutinin mediated autoimmune hemolytic anemia. Blood Rev 2012;26:107115. https://doi.org/10.1016/j.blre.2012.01.002.

20. Silberstein LE, Berkman EM, Schreiber AD. Cold hemagglutinin disease associated with IgG cold-reactive antibody. Ann Intern Med 1987;106:238-242. https://doi. org/10.7326/0003-4819-106-2-238.

21. Gertz MA. How I treat cold agglutinin hemolytic anemia. Clin Adv Hematol Oncol 2019;17:338343.

22. Berentsen $S$, Randen U, Vågan $A M$, Hjorth-Hansen H, VikA, Dalgaard J, et al. High response rate and durable remissions following fludarabine and rituximab combination therapy for chronic cold agglutinin disease. Blood 2010;116:3180-3184. https://doi.org/10.1182/blood-2010-06-288647.

23. Courville KA. A 55 -year-old man with cold agglutinin disease. J Emerg Nurs 2020;46:102104. https://doi.org/10.1016/j.jen.2018.11.016. 